\title{
Ueber die Fortpflanzung einiger Amadinen in der Gefangenschaft.
}

\author{
Von \\ Dr. Garl Bolle.
}

Die Amadinen sind, wie man weiss, eine aus ziemlich zahlreichen Species bestehende Gruppe der grossen Fringillenfamilie, die ausschliesslich auf die Tropenländer der alten Welt beschränkt ist, von der sich aber mehre Arten, in Menge nach Europa gebracht, unserer Beobachtung als Stubenvögel darbieten. Ich will hier hauptsächlich nur von drei Species reden, die nach einem gemeinsamen Typus gebildet, in Körpergestalt, Lebensweise und Stimme eine sehr grosse Uebereinstimmung zeigen. Es sind dies Amadina cantans, malabarica und fasciata.

Im System möchte die Stelle dieser Vögel am Passendsten zwischen den Sperlingen und Kernbeissern gesucht werden. Die, allerdings schwächere, Schnabelbildung reiht sie mehr diesen; der namentlich durch eine horizontale Haltung des Leibes und kurze Beinchen bedingte Habilus, Nistweise und grosse Liebe zur Geselligkeit mehr jenen an. Swainson und Hartlaub placiren sie in die Unterfamilie der Coccothraustinae. Ihnen eigenthümliche Charactere sind: ein keilförmiger Schwanz dessen beide Mittelfedern meist deutlich verlăngert erscheinen und ein ganz besonderer, anhaltender und leiser, bei geschlossenem Schnabel wie aus tiefsler Kehle hervorgegurgelter Gesang. Das Gefieder liegt knapp und schmuck an. Ihr Benehmen ist ein Muster von Friedfertigkeit und Zärtlichkeit der Individuen gegen einander. Was die Nahrung betrift, so geniessen sie vorzugsweis mehlige Sämereien und scheinen daher in ihrem Vaterlande am meisten auf die so unendlich zahlreichen Hirsegräser (Panicum), wohl auch auf die Amaranthus-Arten, der heissen Zone angewiesen zu sein. Ihre Diät in der Voliere besteht aus Hirse und Canariensamen, mit gelegentlichem Zusatz von etwas Grünem; an oliges Gesăme, wie Hanf und Mohn gehen sie nicht leicht.

Die Amadinen erfreuen uns nicht nur durch ihr schmuckes, vertràgliches Wesen und bleiben, bei nur wenig kostspieligem Futter und einigermaassen sorgsamer Pflege, viele Jahre hindurch unsere treuen Stubenkameraden; sie pflanzen sich auch in dem Klima des nördlichen Deutschlands fort und wären, meiner Meinung nach, mit der grössten Leichtigkeit vollständig zu domesticiren. Wie seltsam, dass bisher der Canarienvogel der einzige Singvogel geblieben ist, dessen dauernde Eroberung der Mensch gemacht hat! Hier sind andre - zwar an Virtuositát des Gesanges jenem weit nachstehende - sonst aber nicht minder schöne 
und liebenswürdige Finken, die nur darauf warten, sich uns anzuschliessen, um ein Paar Naturlaute der Tropenwildniss an unseren nordischen Heerd zu tragen. Jetzt zwar liefert sie uns der Handel so leicht und zahlreich, dass das Bedürfniss, sie selbst zu ziehen, weniger fühlbar wird. Aber darin können Aenderungen eintreten. Sind wir schon über alle Gefahr eines Krieges hinaus? Kann die Eifersucht der Seemächte gegeneinander nicht einmal aufs Neue - wer weiss auf wie lange - die Häfen dem überseeischen Verkehr schliessen? Immer noch der kleinste Uebelstand unter vielen wäre es, wenn dann keine exotischen Vögel mehr zu uns gelangten. Dennoch hat Vieillot, und sicher nicht er allein, zur Zeit der Continentalsperre darüber geseufzt und die Zeitgenossen Büffons, die sie ein halbes Jahrhundert früher in Menge besassen, ihretwegen beneidet.

Amadina cantans (Loxia L. et Gmel. Uroloncha Cab.) ist jenes bewegliche, oben gelbbraune, unten hellere Vögelchen mit dem bläulichen Schnabel, das die Händler bei uns „Silberbekchen" oder „Silberfasänchen “, die Franzosen „Bec de plomb" nennen, und das von der Westküste Afrikas, namentlich aus Senegambien, in nicht unbedeutender Menge zu uns gebracht wird. Sein Vaterland scheint über einen grossen Theil des äquatorialen Afrikas ausgedehnt. Alfred Brehm fand es in den grasreichen Steppen des östlichen Sudans, wo es - ein neuer Beweis seiner Zutraulichkeit gegen den Menschen - die Nähe der Nomadenlager aufsucht. Cantans, das Singende, hat es Vater Linné wohl weniger des Wohlklanges und der Stärke, als der langanhaltenden Dauer seines mit ungemeinem Fleisse vorgetragenen Liedchens wegen genannt, das leis und zwitschernd, die gleichfalls zwitschernden häufig ausgestossenen Locktöne des Vogels unterbricht. Die Thierchen lieben es, paarweis oder zu Mehren, dicht aneinander geschmiegt auf der Stange oder dem Aste zu sitzen. Ihre kurzen Flügel gestatten ihnen in der Heimath wohl keinen weiten oder besonders hohen Flug : dafür schlüpfen sie mit der Behendigkeit einer Maus durch das Gezweig. Am Boden werden sie mit schief nach oben gerichtetem Schwänzchen hiipfend gesehn. Man kann die Geschlechter und Altersstufen nur schwer von einander unterscheiden: selbst dass die Mänchen eine gelbere Kehle und Vorderbrust haben sollen, ist ein oft trügliches Kennzeichen. Nicht nur die Gepaarten, auch einander ganz Fremde und sogar Vögel gleichen Geschlechts schliessen sich eng an einander an und scheinen vom ersten Augenblick ihres Beisammenseins unzertrennlich. Auseinandergethan, rufen sie sich mit ängstlich zirpenden, zuletzt scharf und ungeduldig klingenden Tönen. Sie degorgiren nicht; haben aber eine eigenthümliche Weise sich zu 
schnäbeln, die der der Tauben gleicht; putzen sich unter einander auch, ganz wie diese, beständig das Gefieder, zumal am Kopfe. In dem Momente, der der höchsten Extase vorangeht, hebt sich das Hähnchen mehrmals auf den Tarsen senkrecht in die Höhe und fällt ebenso schnell wieder in seine gewöhnliche Stellung zurück: es knikst so zu sagen Es ist dies ein höchst origineller Paarungsgestus, den die Amadinen vor allen andern Vögeln voraus zu haben scheinen. Die Begattung wird dann entweder auf einem Aste oder, häufiger noch, im Innern des Nestes, vollzogen. „Die Wärme unsrer Sommer, sagt Vieillot in seinen „Oiseaux chanteurs" genügt zu ihrer Vermehrung in Europa; da sie aber oft schon Ende Winters zu legen anfangen, so thut man, um sicher zu gehen, wohl, die Bruten bis zum Mai hinaus zu schieben. Sie nisten vom Februar bis August."

Bei einer anzulegenden Hecke dieser Vögel hat man darauf Rücksicht zu nehmen, dass es entschiedene Höhlenbrüter sind. Sie bauen sowohl in Höhlungen mit sehr weitem Eingang, als auch in Brutkästchen mit ganz engem Loche und übernachten, wenn man ihnen, was stets geschehen sollte, dergleichen hinhängt, viel lieber darin, als dass sie auf einer Sprosse sitzend schliefen. Nichts ist reizender, als ein gemeinsames Lager solcher Thierchen zu belauschen und sie ihre Köpfe neugierig aus einem Astloche hervorstrecken zu sehen. Die respective Mittheilung der animalischen Wärme in einem geschlossenen Raume erleichtert ihnen, abgesehen davon, dass sie ihrer Natur gemäss ist, auch das Ertragen kühlerer Nâchte. Einen ganz besonders merkwïrdigen Umstand erblicke ich darin, dass allein das Männchen der Baumeister ist: nie habe ich das Weibchen auch nur einen Halm zutragen sehen! Es begnügt sich damit, passiv im Neste oder vor demselben silzend, die Huldigungen seiner fleissigeren Hälfte entgegen zu nehmen. Diese Eigenthümlichkeit stellt die drei uns hier beschâftigenden Amadinen in psychischer Hinsicht auf die höchste Stufe unter den Fringillen; denn nirgend tritt das Gefühl der elterlichen Liebe so stark und fürsorglich bei einem Geschlechte auf, welches es sonst mit den ehelichen Pflichten leichter zu nehmen pflegt.

Ist die Hŏhle weit, so wird sie mit einem enormen Wust von Nistmaterial angefült. Alles ist dem Vogel dazu recht: Heu, Moos, Baumwolle, Zwirn, Papierstückchen, ja selbst frisches Grün, wie ich ihn denn mehrmals mit Vogelmiere bauen sah. Fällt zu viel Licht hinein oder war das Nest in einem nur durch Gitterwerk geschlossenen Harzer Bauerchen angelegt, so wird es uherwölbt und nur vorn und oben eine ganz kleine Oeffnung zum Hineinschlüpfen gelassen. In einem Nist- 
kästchen mit engem Eingange wird dagegen nur der Boden mit möglichst weichen Stoffen gepolstert und die Oeffnung ein wenig verbaut. Die Arbeit des Bauens begleitet der Vogel mit einem ganz eignen, immerwährenden Gezwitscher. Die Durchschniltszahl der kleinen und länglichen, schneeweissen Eier beträgt vier. Diese werden 10-11 Tage bebrütet und jetzt spielt das Weibchen nicht mehr die Rolle der Emancipirten, sondern bedeckt sie abwechselnd mit dem Männchen. Bei jedem lauten Geräusch streichen sie vom Neste, um bald darauf vorsichtig auf dasselbe zurückzukehren.

Ich hatte bereits 1855 ein Cantans-Pärchen bei meinem Freunde, dem Dr. L. Tichy in Berlin nisten sehen. Es war zwischen Winter und Frühling. Man halte ihnen ein mit Tannenzweigen ausgeschmücktes Bauer am Fenster eines geheizten Zimmers angewiesen; aber, mit ihrer Eigenschaft als Höhlenbrüter unbekannt, ihnen nur offne Körbchen gegeben. Diese suchten sie so viel als möglich zu überwölben; baueten wochenlang; vertauschten dann, unzufrieden, das eine Nest mit dem andern und zuletzt ward, nach Legung des ersten Eis, das Weibchen todt auf demselben gefunden.

Im Sommer 1858 ist mir, der ich zum ersten Male diese Vögel beisammen hielt, der Versuch geglückt. Sie hatten vom April an dreimal vergeblich gebaut und Eier gelegt. Diese Eier verschwanden plötzlich oder wurden zertrümmert vorgefunden. Ich habe eine die Voliere mit ihnen zugleich bewohnende Fringilla nitens in starkem Verdacht der Eierfresser gewesen zu sein. Die gelungene Brut ward in einem Nistkästchen, das ihnen vor andern Vögeln Ruhe gewährte, zu Ende geführt. Von Schwäche oder Kranksein des Weibchens nach dem mehrmaligen Legen war keine Rede gewesen. Die Jungen sind anfangs fast ganz kahl und sehr hässlich: schwärzlich roth mit kleinem, breitem Schnabel und weiter Mundöffnung, in der eine gleichfalls breite, gelbgesäumte Zunge liegt. Auch die Mundwinkel sind gelb. Diese Thierchen sperren fortwährend und werden von den Eltern aus dem Kropfe mit geschälter Hirse gefüttert, deren Körnchen man bei den Heranwachseuden in dem durchsichtigen Kropfe liegen sieht. Die ersten anderthalb Wochen ihres Lebens entwickeln sie sich langsam; nachher um so schneller. Mit zunehmendem Alter, nehmen sie, die lange nackt bleiben, eine bläuliche Färbung an: man möchte sie dann eher für kleine ekelhafte Amphibien als für Vögelchen ansehen. Anfangs halten die Alten das Nest rein: bald jedoch wächst ihnen diese Arbeit über den Kopf. Es wird dann von den grösserwerdenden Jungen in eine wahre Cloake verwandelt und riecht so abscheulich wie die verrufene Nisthöhle des 
Wiedehopfs. Sich selbst aber bewahren nichts destoweniger die Kleinen vollkommen sauber. Um dies zu bewirken, sitzen sie mit senkrecht uach oben gekehrtem Hinterleibe und perpendikulärem oder etwas vorn übergebogenem Schwänzchen in der allerseltsamsten Positur, auf Brust und Kehle ruhend, im Neste und vermeiden so eine jede Berührung mit den von Schmutz starrenden Wänden, gegen welche sie ihre Exkremente hoch nach oben schleudern. Mit eben dem Erfolge beweisen Vater und Mutter die Wahrheit des Wahlspruchs: Puris omnia pura. Sobald sie hineinschlüpfen, um zu füttern, erhebt sich drinnen ein Gezwitscher, wie von jungen Sperlingen. Ameiseneier, die sie sonst wohl fressen, wurden behufs der Fütterung der Jungen ganz verschmäht; desgleichen Grünes. Man kann daher Cantans und seine Vettern zu den ausschliesslichsten Körnerfressern unter den Finken zählen, von denen die meisten wenigstens ihre kleinen Jungen mit Insectenkost atzen.

Meine Silberbekchen waren am 11. Juli, 19 Tage alt, fast flügge. Zwei Tage darauf, am 21, verliess das Aelteste das Nest, kehrte jedoch bald wieder dahin zurück, um noch einen Tag lang mit seinen $\mathrm{Ge}-$ schwistern darin sitzend zu verharren. Dann flogen Alle auf einmal aus, jedoch nicht, ohne sich in den nächstfolgenden Tagen noch täglich stundenlang in ihre Geburtshöhle oder, nachdem ich diese zum Zwecke gründlicher Reinigung weggenommen, in eine andre ihr ähnliche zurückzuziehen und darin, wenn man sie öfnete, ebenso fest, wie vor dem Flüggewerden, liegen zu bleiben. Sie nestelten sich dann, wie junge Zaunkönige eng zusammen. Die Ausgeflogenen sitzen gleich aufmarschirten kleinen Soldaten neben einander auf der Stange und fordern unter lautem Zirpen, von Zeit zu Zeit mit aufgesperrtem Schnabel, ihre Nahrung von den Alten. Jedes sucht den Mittelplatz zu gewinnen und springt deshalb mitunter den Andern auf den Rücken. Vater und Mutter füttern gleich eifrig: ersterer, wie mir schien, noch etwas emsiger als die schon wieder dem Vorgefühl einer neuen Brut lebende Gattin. Sehr niedlich sieht es aus, wenn eins der Eltern hochaufgerichtet über die Front machenden Jungen hinweglangt, um auch dem Letzten am entgegengesetzten Ende seinen Antheil einzustopfen. Fünf und zwanzig Tage alt, sah ich sie zum erstenmal allein fressen und zwar gleich Hirse und Canariensamen. Von weichem Futter nahmen sie gar keine Notiz.

Beide Gatten würden, nach der erwähnten, wahrscheinlich noch eine Brut vor der Mauser gemacht haben, hälte nicht eine unaufschiebbare Reise ihnen meine pllegende Gegenwart entzogen.

Ich hatte vorher schon, ehe ich wusste, wie gut sie brüten, versucht ihre Eier Canarienvŏgeln unterzulegen; erzielte jedocb keine gün- 
stigen Resultate. Die Jungen kamen zwar aus; ich fand sie aber schon am zweiten Tage von ihrer zu schweren Pflegemutter erdrückt. Mit glücklicherem Ausgange dürfte dies Experiment mit Eiern der grösseren Amadina fasciata wiederholt werden.

Amadina cantans nistet in Käfichen von mittlerer Grösse ebenso gern als in fliegenden Hecken. Da sie sehr verträglich gegen andre Vögel sind und ihrerseits von diesen in ihren Troglodyten-Wohnungen kaum je beunruhigt werden, so kann man sie den Canarienvögeln als Stubenbürschchen ohne Schaden zugesellen. Die einzelne Brut auf 35 Tage berechnet, würden sie unter günstigen Umständen jährlich fünf Bruten zu erziehen im Stande sein.

Wie ein von mir im vergangnen Sommer angestellter Versuch beweist, ist nichts leichter als zwischen A. cantans und malabarica $\mathbf{L}$. Bastarde zu ziehen. Beide Vögel sind an Grösse, Gestalt, Farbe und Stimme einander so ähnlich, dass sie von Unkundigen, auch von den meisten Vogelhändlern, für ein und dieselbe Art angesehen werden, um so mehr, da auch ihre Sitten fast vollkommen übereinstimmen. Malabarica ersetzt die afrikanische Cantans in Ostindien. Damit man die seltner lebend zu uns gelangende malabarica, die sogar in Museen öfter unrichtig bestimmt steht, von der bekannteren Species unterscheiden könne, will ich ihre Beschreibung hiehersetzen. Der Vogel ist von gleicher Grösse oder um einen Gedanken stärker als cantans: am Oberkörper chocoladenbraun, am Oberkopf mehr braun. Der Bürzel ist weiss; der ganze Unterleib nebst den beim Silberbekchen mit Kehle und Oberbrust dunkel isabellgelben Backen, weisslich, an den Seiten mit zarten chamois Mondfleckchen. Schwingen und Schwanz sind schwärzlich : letzterer mit schön purpurnem Schimmer. Die zwei miltelsten Schwanzfedern sind, wie bei cantans, pfeilförmig verlängert und überragen die Uebrigen sehr sichtlich: Kennzeichen der Gattung Uroloncha Cabanis. Der Schnabel ist bläulich; die Füsse sind bläulich fleischfarben. In Silten, Nahrung und Gesang identificirt sich dieser Vogel fast mit dem vorigen. Auch gesellt er sich zu Vögeln jener Art mit derselben Leichtigkeit und nicht minder zärtlich, wie zu seines Gleichen.

Die Bastarde, die ich erhielt, waren im Nestkleide oben chocoladenbraun mit schwärzlich geschupptem Oberkopfe. Der weisse Bürzel fehlte ihnen; auch verriethen Kehle, Brust und Halsseiten durch gelbere Färbung ihre mütterliche Abstammung. Der Schnabel war bleigrau, die Füsse zart fleischfarb. Kurze Zeit nach der Brut starb das Weibchen während meiner Abwesenheit. Das Männchen und die Jungen besitze ich noch. Letztere sind jetzt dem Vater sehr ähnlich, zeichnen sich 
jedoch vor ihm immer noch durch einen gelben Anflug an Kehle und Oberbrust, hauptsächlich aber durch einen schwarz und weisś gescheckten Bürzel aus, an dem jede einzelne Feder schwarz mit langer, weisser Spitze ist. Bei zwei von ihnen tragen höchst merkwürdiger Weise ausserdem noch die hintersten etwas verlängerten Bürzelfedern deutlich rosenrothe Spitzen.

Die dritte Amadina, der wir hier Aufmerksamkeit widmen, ist $\boldsymbol{A}$. fasciata Hartl.; Loxia Gmel.; Fringilla detruncata Licht.; der Bluthals oder Bandvogel; Bechsteins gebänderter Kernbeisser; die Gorgecoupée oder Collerette der Franzosen; ein bedeutend grösserer Vogel als die beiden Vorigen, gedrungen, ja eher etwas plump gebaut, aber durch die schuppige Zeichnung des zimmtfarbnen Gefieders, die beim Männchen von einem blutrothen Kehlband und einem rothbraunen, an das des Rebhuhns erinnerndem Brustschild gehoben wird, nichtsdestoweniger eine gefällige Erscheinung. Wer sie zuerst sieht, wird unwillkührlich an eine durchschnittene frisch blutende Kehle gemahnt; fast wie einst bei der vollendeten Schönheit Anna Boleyn blutrothes Geäder um den zarten Hals das bevorstehende tragische Schicksal dieser unglücklichen Königin vorausgesagt haben soll. - Es ist dies, nach dem Reisvogel, vielleicht der verbreitetste Exot in den europäischen Volieren: man trift ihn tief im Binnenlande, wie ich ihn u. a. selbst in Süddeutschland und auf dem Vogelmarkte von Mailand sah. Er bildet auch in der That ein Hauptkontingent zu jenem unschuldigen Sklavenhandel, der von der afrikanischen Westküste ausgeht und muss, der zu uns gebrachten ladividuenzahl nach zu urtheilen, einer der häufigsten Senegalfinken sein. In N. 0 . Afrika erreicht er den 14. Grad N. Br. Zwar wird er unter den Vögeln des Sudan-Urwaldes sowohl als der Steppe mit aufgeführt; doch dürfte er vielleicht an gewissen Localitäten auch in den Wohnungen des Menschen angesiedelt gefunden werden, und namentlich, wenn anders wir es wagen können, ein Citat Barths auf ihn zu beziehen, selbst das Innre der so lange mysteriös gebliebenen Hauptstädte Agades und Timbuctu freiwillig bewohnen. Der berühmte Reisende sagt:

"Ich erfrente mich der Gesellschaft einer kleinen niedlichen Art von Finken, welche in grosser Anzahl alle Zimmer in Agades heimsuchen und dort ihre Nester bauen, ganz ebenso wie in dem, in allen Beziehungen Agades so schwesterlich zur Seite stehenden Timbuctu. Namentlich ist das Mănnchen mit seinem rothen $\mathrm{Halse}$ überaus niedlich. Die Jungen wurden Ende October gerade flügge. ${ }^{\star}$ Diese kurze Charakteristik scheint mir weniger auf die zaunkönighaft kleine Estrelda minima, den verbreitetsten sudanischen Hausastrild, als auf Amadina 
fasciata zu passen. Und ist doch auch minima zugleich in den niederen, dornigen Gebüschen der Urwälder und in Gebäuden heimisch.

Sitten und Nahrung kommen mit denen des Silberbekchens überein, nur sind hier die knicksenden Bewegungen des Hähnchens während der Liebesperiode lebhafter und von noch groteskerer Grazie. Dieses singt sein gutturales, dem der Rauchschwalbe ähnelndes Lied, nicht nur bei Tage, sondern auch fleissig Abends, sobald nur ein matter Lichtstrahl das Plätzchen, welches es einnimmt, erhellt. Der beiden Geschlechtern gemeinsame Lockruf ist fast der des Haussperlings, nur etwas schwächer. Auch bei dieser Species zeigen die Gatten eine musterhafte Anhänglichkeit aneinander; auch hier begegnen wir derselben Unzertrennlichkeit, denselben Liebkosungen und dem gleich exclusiven Bautriebe des Männchens; aber ein leidenschaftlicheres Temperament und die Heftigkeit von oft nichts weniger als platonischen Wallungen verleitet den Bluthals nicht selten dazu, seinem Weibchen übel zu begegnen, wenn dasselbe sich den Anforderungen seiner Sinnlichkeit nicht unbedingt fügen will. Ich sah ihn demselben Gewalt anthun, zu einer Zeit, wo es, kränkelnd, sich nur nach Ruhe sehnte. Ueber den vollstăndigen Verlauf der Fortpflanzung dieses Vogels besitze ich weniger genaue Erfahrungen. Es steht jedoch fest, dass seine Heckzeit sich auf den grössten Theil des Jahres erstreckt: ja sie erleidet, bei gehöriger Wărme, eigentlich nur durch die im August stattfindende Mauser eine Unterbrechung. Ich sah Bluthälse auf den canarischen Inseln sich mit der grössten Leichtigkeit in der Gefangensshaft vermehren; auch in Deutschland ist ihr Hang hierzu ein fast unwiderstehlicher. Beim Männchen manifestirt er sich vorzugsweise. Dies sitzt, lange vor dem Weibchen, stundenlang in der Nisthöhle, die einen recht engen Eingang haben muss, der später inwendig so viel als möglich verschlossen wird. Innen häuft dann das Hähnchen ein aus trocknen Halmen und Pflanzenstengeln, nie aus weichen und warmen Stoffen gebildetes, schlichtes Lager an, auf welches $3-4$ weisse Eier gelegt werden. Die Weibchen dieser Species scheinen mir weniger emsige Brüterinnen, als die von cantans zu sein; vielleicht, weil sie beim Legen mehr leiden. Wenn man ihnen nicht recht reichlich Kalk und Eierschaalen in den Käfig wirft, bringen sie leicht Fliesseier zur Welt, die dann stets die Wehen zu einer lebensgefährlichen Krise machen. Mir sind mehre Weibchen nach einander in Folge schweren Legens zu Grunde gegangen oder haben das Männchen beim Brüten später nicht ausdauernd genug unterstützen können. Die verwittweten männlichen Bluthälse gerathen durch den Verlust ihrer Gattinnen ausser sich. Sie, die sonst im besten Einverständniss mit 
Vögeln andrer Art lebten, werden vereinsamt, plötzlich so bissig und unleidlich, dass man genöthigt ist, sie allein zu sperren.

Wer auf die Vermehrung dieses Vogels im Zimmer bedacht ist, versehe sich daher mit einer grösseren Zahl Weibchen als Männchen, um etwaige Verluste ohne Zögerung ersetzen zu können. Auch bemühe man sich, den nistenden Bluthälsen eine möglichst hohe Temperatur zu verschaffen. Nur unsre wärmsten Sommermonate dürften sie ihnen genügend darbieten; leichter wohl noch im Winter die unmittelbare Nähe des Ofens. Vom Januar an, kann man mit Sicherheit auf Lust zum Nisten ihrerseits rechnen. Diese äussert sich selbst in ganz kleinen Käfigen, wenn nur ein Nistloch darin angebracht werden kann.

Noch eine Amadine, die sich ebenfalls ohne grosse Hindernisse als Stubenvogel vermehren soll, ist A. erythrocephala L., Loxia brasiliana Gmel., aschgrau-schuppig mit scharlachrothem Kopfe, dem Typus der Paroaria's analog gefärbt. Da dieselbe jedoch nur höchst selten lebend zu uns kommt, übergehe ich sie. Man wird sie - einen Vogel Angola's - in Lissabon, wo ich sie allein sah, besser kennen, als in Berlin.

Die Zucht der Amadinen dürfte durch die verhältnissmässig geringen Schwierigkeiten, welche sie darbietet, wohl dazu auffordern, allgemeiner und in grösserem Maassstabe betrieben zu werden. Abgesehen von dem Vergnügen, welches sie gewährt, könnte dieselbe, bei der grossen Nachfrage nach den beliebten Vögelchen, deren Preis für das Paar jetzt zwischen 3 und 4 Thlrn. schwankt, ärmeren, an das Haus gefesselten Personen einen nicht unbedeutenden kleinen Nebenverdienst abwerfen, der nicht, wie bei den Canarienvögeln durch die Kostspieligkeit des Eierfutters der Jungen geschmälert würde.

Berlin, am 30. November 1858.

\section{Miterarische Berichte.}

Denkwurdigkeiten einer Reise nach dem russischen Amerika, nach Mikronesien und durch Kamtschatka von F. H. von Kittlitz. Gotha 1858.

Im Jahre 1825 verliess ein junger preussischer Officier die glänzende Carriere der Waffen, um sich als Naturforscher einer von Russland ausgehenden Expedition anzuschliessen, die im Begriff stand, eine Reise um die Welt anzutreten. Ornithologie war von jeher das Lieb- 


\section{$2 \mathrm{BHL}$ Biodiversity Heritage Library}

1859. "Ueber die Fortpflanzung einiger Amadinen in der Gefangenschaft." Journal

$f u$

r Ornithologie 7, 37-45. https://doi.org/10.1007/bf02007569.

View This Item Online: $\underline{\text { https://www.biodiversitylibrary.org/item/50195 }}$

DOI: https://doi.org/10.1007/bf02007569

Permalink: https://www.biodiversitylibrary.org/partpdf/141437

\section{Holding Institution}

Natural History Museum Library, London

\section{Sponsored by}

Natural History Museum Library, London

\section{Copyright \& Reuse}

Copyright Status: Public domain. The BHL considers that this work is no longer under copyright protection.

This document was created from content at the Biodiversity Heritage Library, the world's largest open access digital library for biodiversity literature and archives. Visit BHL at https://www.biodiversitylibrary.org. 